\title{
Stability of sets for impulsive differential systems
}

Giovanni Matarazzo, Nikolai Perestyuk, and Olga Chernikova 


\title{
STABILITY OF SETS FOR IMPULSIVE DIFFERENTIAL SYSTEMS
}

\author{
GIOVANNI MATARAZZO, NIKOLAI A. PERESTYUK, AND OLGA S. CHERNIKOVA
}

[Received: April 15, 2004]

\begin{abstract}
Aвstract. The problems of sets' stability for impulsive differential systems are considered. A short survey of the recent results connected with the stability properties of sets for impulsive differential systems is given.
\end{abstract}

Mathematics Subject Classification: 34D20

Keywords: Impulsive differential system, invariant set, integral set, stability of invariant set, stability of integral sets

\section{STABILITY OF INVARIANT SETS FOR DISCONTINUOUS DYNAMICAL SYSTEMS}

In the present paper, we give a survey of the recent results connected with the stability properties of sets for impulsive differential systems and characterise the methods of investigation of the sets' stability.

Let us first consider the system of differential equations (discontinuous dynamical system $[\mathbf{1 , 2}]$ )

$$
\begin{gathered}
\frac{d x}{d t}=f(x), \quad x \notin \Gamma, \\
\Delta x_{\mid x \in \Gamma}=T x-x=I(x),
\end{gathered}
$$

where $x \in \bar{D} \subset \mathbb{R}^{n}, f(x) \in C\left[\bar{D}, \mathbb{R}^{n}\right], D$ is a bounded domain in $\mathbb{R}^{n}, t \in[0, \infty), T$ is an operator, $T: \Gamma \rightarrow Q, \Gamma$ is a surface without contact for the system $\frac{d x}{d t}=f(x)$, $\Gamma \subset \bar{D}$ (possibly, $\Gamma=\partial D$ ), and $Q$ is a closed subset of $\bar{D}$.

We shall assume that the conditions for existence and uniqueness of solutions of (1.1) are fulfilled and also suppose that each solution of (1.1) exists on $[0,+\infty)$. Denote by $x(t)=x\left(t, x_{0}\right)$ the solution of (1.1) satisfying initial condition $x(0)=x_{0}$.

We indicate the following kinds of motions of the system (1.1): $1^{\circ}$ motions without impulse effect; $\mathbf{2}^{\circ}$ motions whose trajectories hit the set $\Gamma$ no more than finitely many times; and $\mathbf{3}^{\circ}$ motions whose trajectories hit the set $\Gamma$ infinitely many times. Let $\Omega$ be the set of such points $x_{0}$ that trajectories $\left\{x\left(t, x_{0}\right): t \in[0,+\infty)\right\}$ meet $\Gamma$ infinitely many times. Let us put $S_{\delta}(M)=\{x \in \bar{D}: \varrho(x, M)<\delta\}$ and $U_{\delta}(M)=$ $\{x \in \bar{D}: 0<\varrho(x, M)<\delta\}$ for any set $M \subseteq \bar{D}$. 
We shall say that a set $M \subseteq \bar{D}$ is an invariant set of system (1.1) if $x_{0} \in M$ implies that $x\left(t, x_{0}\right) \in M$ for $t \geq 0$.

A closed invariant set $M$ of the system (1.1) is said to be stable if for any $\varepsilon>0$ there exists $\delta=\delta(\varepsilon)>0$ such that $x_{0} \in S_{\delta}(M)$ implies $x\left(t, x_{0}\right) \in S_{\varepsilon}(M), t \geq 0$, for any solution $x(t)=x\left(t, x_{0}\right)$ of (1.1).

A closed invariant set $M$ of the system (1.1) is said to be asymptotically stable if $M$ is stable and there exists $\delta_{0}>0$ such that the relation $x_{0} \in S_{\delta_{0}}(M)$ implies that $\lim _{t \rightarrow \infty} \varrho\left(x\left(t, x_{0}\right), M\right)=0$ for any solution $x(t)=x\left(t, x_{0}\right)$ of $(1.1)$.

A closed invariant set $M$ of the system (1.1) is said to be stable (asymptotically stable) with respect to the set $B \subset \bar{D}$ if the solutions beginning in $B$ satisfy the conditions of the definition of stability (asymptotic stability) for the set $M$.

The closed invariant set $M$ is said to be unstable if conditions of the definition of stability fail to hold.

To establish stability results for invariant sets of the system (1.1) we use an auxiliary function $V(x)$ possessing certain properties.

Let $V(x)$ be a function continuously differentiable in $\bar{D}$ and such that

$$
V(x)=0, \quad x \in M_{0}, M_{0} \subset \bar{D}, M_{0} \neq \varnothing ; \quad V(x)>0, x \in \bar{D} \backslash M_{0} .
$$

Denote by $K_{0}$ the class of continuous functions $\varphi: \mathbb{R}_{+} \rightarrow \mathbb{R}_{+}$such that $\varphi(s)=0$ if and only if $s=0$. (1.1)

We formulate some conditions sufficient for the stability of invariant sets of system

Theorem 1 (see [3]). $\quad$ (A) Assume that there exists a function $V(x)$ satisfying condition (1.2) and the conditions

$$
\begin{gathered}
\langle\operatorname{grad} V(x), f(x)\rangle \leq 0 \quad(x \notin \Gamma), \\
V(x+I(x)) \leq V(x) \quad(x \in \Gamma) .
\end{gathered}
$$

Then $M_{0}$ is a stable invariant set for system (1.1).

(B) Assume that there exists a function $V(x)$ satisfying conditions (1.2), (1.4), and

$$
\langle\operatorname{grad} V(x), f(x)\rangle \leq-\varphi(V(x)) \quad(x \notin \Gamma),
$$

where $\varphi(s) \in K_{0}$. Then $M_{0}$ is an asymptotically stable invariant set of the system (1.1).

Let $M_{0}$ be a closed invariant set such that $M_{0} \cap \Omega \neq \varnothing, M_{0} \neq \Omega$.

Theorem 2 (see [3]). If there exists a function $V(x)$ satisfying conditions (1.2), (1.3), and

$$
V(x+I(x))-V(x) \leq-\psi(V(x)), \quad x \in \Gamma,
$$

where $\psi(s) \in K_{0}$, then $M_{0}$ is an asymptotically stable invariant set of system (1.1) with respect to set $\Omega$. 
The proofs of the statements of this theorem are based on reasoning similar to $[1,2,4]$.

The following theorem gives more interesting conditions for stability properties of the invariant set in suggestion that

$$
t_{k+1}^{x_{0}}-t_{k}^{x_{0}} \geq \theta>0, \quad k \geq 1 .
$$

We denote by $t_{k}^{x_{0}}, k=1,2, \ldots$, the moments at which the discontinuous trajectory $x\left(t, x_{0}\right)$ meets the set $\Gamma$.

Theorem 3. Assume that for differential system (1.1), there exists a function $V(x)$ satisfying conditions (1.2), (1.5), and the condition

$$
V(x+I(x)) \leq \psi(V(x)), \quad x \in \Gamma,
$$

where $\psi(s) \in K_{0}$.

(A) If there exists $a_{0}>0$ such that, for all $a \in\left(0, a_{0}\right]$,

$$
\int_{a}^{\psi(a)} \frac{d s}{\varphi(s)} \leq \theta
$$

where $\theta$ is the number from (1.6), then $M_{0}$ is a stable invariant set of the system (1.1).

(B) If there exists $a_{0}>0$ such that, for all $a \in\left(0, a_{0}\right]$,

$$
\int_{a}^{\psi(a)} \frac{d s}{\varphi(s)} \leq \theta-\gamma
$$

for some $\gamma>0$, then $M_{0}$ is an asymptotically stable invariant set of system (1.1).

Consider some conditions for instability of the set $M_{0} \subset \bar{D}[3]$.

Theorem 4. Assume that there exists a function $V(x)$ continuously differentiable on the set $S_{h}\left(M_{0}\right)$ and such that $V(x)=0, x \in M_{0}$ and, for any $\delta(0<\delta<h)$, $U_{\delta}\left(M_{0}\right) \cap \Pi \neq \varnothing$, where $\Pi=\{x \in \bar{D}: V(x)>0\}$. Assume also that, on the set $\Pi$, the function $V(x)$ satisfies the conditions

$$
\langle\operatorname{grad} V(x), f(x)\rangle \geq \varphi(V(x)) \quad(x \notin \Gamma)
$$

and

$$
V(x+I(x)) \geq V(x) \quad(x \in \Gamma)
$$

where $\varphi(s) \in K_{0}$. Then $M_{0}$ is an unstable set for system (1.1).

Theorem 5. Assume that there is a continuously differentiable function $V(x)$ defined on the set $S_{h}\left(M_{0}\right)$ such that $V(x)=0, x \in M_{0}$, and for any $\delta(0<\delta<h)$, $U_{\delta}\left(M_{0}\right) \cap \Pi \neq \varnothing$, where $\Pi=\{x \in \bar{D}: V(x)>0\}$. Assume also that, on the set $\Pi$, the function $V(x)$ satisfies the conditions

$$
\langle\operatorname{grad} V(x), f(x)\rangle \geq \varphi(V(x)), \quad(x \notin \Gamma)
$$


and

$$
V(x+I(x)) \geq \psi(V(x)) \quad(x \in \Gamma),
$$

where $\varphi(s) \in K_{0}, \psi(s) \in K_{0}$.

If for any $a \in\left(0, a_{0}\right]$, where $a_{0}=\sup _{x \in \Pi} V(x)$, and some $\gamma>0$

$$
\int_{\psi(a)}^{a} \frac{d s}{\varphi(s)} \leq \theta-\gamma,
$$

then $M_{0}$ is an unstable set for system (1.1).

Analogous results may be established in the case when the sequence $\left\{t_{k}^{x_{0}}\right\}, k=$ $1,2, \ldots$, satisfies the condition $t_{k+1}^{x_{0}}-t_{k}^{x_{0}} \leq \theta_{1}$.

The results above are natural generalisations of the stability results for impulsive systems with unique equilibrium positions. If the invariant set of system (1.1) is a point $x=0$, then the results mentioned above give the well-known sufficient conditions for stability, asymptotic stability, and instability of the trivial solution (see the survey of the corresponding results in [5]).

\section{Stability Of INTEgRal SETS OF THE Differential SYSTEMS WITH IMPULSIVE}

\section{PERTURBATIONS}

Consider now the impulsive differential system

$$
\begin{gathered}
\frac{d x}{d t}=f(t, x), \quad t \neq t_{i}(x), \\
\left.\Delta x\right|_{t=t_{i}(x)}=I_{i}(x),
\end{gathered}
$$

where $x \in \mathbb{R}^{n}, t \in \mathbb{R}_{+}, f(t, x), I_{i}(x), i=1,2, \ldots$, are continuous vector-functions, $t_{i}(x)$ are continuous functions such that $0<t_{1}(x)<t_{2}(x)<\ldots, x \in \mathbb{R}^{n}, t_{i}(x) \rightarrow \infty$ as $i \rightarrow \infty$ uniformly in $x \in \mathbb{R}^{n}$. We assume that, for any point $\left(t_{0}, x_{0}\right) \in \mathbb{R}_{+} \times \mathbb{R}^{n}$, there exists a unique solution $x(t)=x\left(t, t_{0}, x_{0}\right)$ of $(2.1)$ on $\left[t_{0}, \infty\right)$ satisfying $x\left(t_{0}\right)=x_{0}$, and that the integral curve of each solution meets each of the hypersurfaces $t=t_{i}(x)$ only once, i. e., the phenomenon of "beating" is not observed. Sufficient conditions for the absence of the phenomenon of "beating" are well-known (see, e. g., $[\mathbf{1 , 2}, \mathbf{6}]$ ).

We recall $[1,2]$ that any solution $x\left(t, t_{0}, x_{0}\right)$ of $(2.1)$ is a piecewise continuous function with points of discontinuity $\theta_{i}^{x}, \theta_{i}^{x}=t_{i}\left(x\left(\theta_{i}^{x}\right)\right), i=1,2, \ldots$;

$$
\begin{aligned}
x\left(\theta_{i}^{x}, t_{0}, x_{0}\right) & =x\left(\theta_{i}^{x}-0, t_{0}, x_{0}\right), \\
x\left(\theta_{i}^{x}+0, t_{0}, x_{0}\right) & =x\left(\theta_{i}^{x}, t_{0}, x_{0}\right)+I_{i}\left(x\left(\theta_{i}^{x}, t_{0}, x_{0}\right)\right) .
\end{aligned}
$$

We shall say that set $M \subset \mathbb{R}_{+} \times \mathbb{R}^{n}$ is an integral set of system (1.1) if, for any point $\left(t_{0}, x_{0}\right) \in M$, it follows that $\left(t, x\left(t, t_{0}, x_{0}\right)\right) \in M$ for $t \geq t_{0}$.

Note that, together with a point $\left(t^{\bullet}, x^{\bullet}\right) \in M$ belonging to the surface of discontinuity $\left(t^{\bullet}=t_{i}\left(x^{\bullet}\right)\right)$, the set $M$ contains also the point $\left(t^{\bullet}, x^{\bullet}+I_{i}\left(x^{\bullet}\right)\right)$. 
For each $i=1,2, \ldots$, we denote by $\Lambda_{i}$ the set of time moments when the integral curves belonging to $M$ meet the hypersurface $t=t_{i}(x)$, i. e, the set of solutions of the equation $\tau=t_{i}(x(\tau)),(\tau, x(\tau)) \in M$. If, for instance, the integral set is the integral curve $\left\{(t, x): t \in \mathbb{R}^{+}, x=x\left(t, 0, x_{0}\right)\right\}$ then $\Lambda_{i}$ consists of a single point: $\Lambda_{i}=\left\{\theta_{i}^{x}\right\}$. In the case where $t_{i}(x) \equiv \tau_{i}, i=1,2, \ldots$, each set $\Lambda_{i}$ also consists of a single element: $\Lambda_{i}=\left\{\tau_{i}\right\}$

Let $M(t)=\left\{x \in \mathbb{R}^{n}:(t, x) \in M\right\}$. If $t=\theta_{i} \in \Lambda_{i},\left(\theta_{i}, x\right) \in M$ and $\theta_{i}=t_{i}(x)$, then, as it was noted above, the set $M$ contains both points: $\left(\theta_{i}, x\right)$ and $\left(\theta_{i}, x+I_{i}(x)\right)$ and, therefore, the section $M\left(\theta_{i}\right)$ contains both points $x$ and $x+I_{i}(x)$.

For $\theta_{i} \in \Lambda_{i}$, we distinguish two subsets of the section: $M\left(\theta_{i}\right)$ and $M_{i+1}\left(\theta_{i}\right)$. The properties that a point $x \in \mathbb{R}^{n}$ belongs to $M\left(\theta_{i}\right)$ or $M_{i+1}\left(\theta_{i}\right)$ are defined in the following way. Let $\Omega_{i}=\left\{(t, x) \in \mathbb{R}_{+} \times \mathbb{R}^{n}: t_{i-1}(x)<t \leq t_{i}(x)\right\}, i=1,2, \ldots, t_{0}(x) \equiv 0$. If $\left(\theta_{i}, x\right) \in M \cap \Omega_{i}$, then $x \in M_{i}\left(\theta_{i}\right)$; if $\left(\theta_{i}, x\right) \in M \cap \Omega_{i+1}$ or $\left(\theta_{i}, x\right)$ is a boundary point for $M \cap \Omega_{i+1}$ then $x \in M_{i+1}\left(\theta_{i}\right) ; M\left(\theta_{i}\right)=M_{i}\left(\theta_{i}\right) \cup M_{i+1}\left(\theta_{i}\right),\left(\theta_{i} \in \Lambda_{i}\right)$. It is possible that $M_{i}\left(\theta_{i}\right) \cap M_{i+1}\left(\theta_{i}\right) \neq \varnothing$.

Assume that $M(t) \neq \varnothing, M(t) \subseteq Q \subset \mathbb{R}^{n}, t \in \mathbb{R}_{+}$, where $Q$ is a compact set. Furthermore we shall suppose that $Q_{\varepsilon_{0}} \subset B_{h}$, where $Q_{\varepsilon_{0}}=\left\{x \in \mathbb{R}^{n}: \varrho(x, Q)<\varepsilon_{0}\right\}$, $B_{h}=\left\{x \in \mathbb{R}^{n}:\|x\| \leq h\right\}$, for some $\varepsilon_{0}>0, h>0$.

In the case when impulsive system (1.1) is a system with impulses at fixed times, i. e., $t_{i}(x) \equiv \tau_{i}, i=1,2, \ldots$, we differentiate two subsets of the section of integral set $M$ only for points $\tau_{i}$ : if $\left(\tau_{i}, x\right) \in M \cap \Omega_{i}$, then $x \in M_{i}\left(\tau_{i}\right)$; if $\left(\tau_{i}, x\right) \in M$ and $\left(\tau_{i}, x\right)$ is a boundary point of $M \cap \Omega_{i+1}$, then $x \in M_{i+1}\left(\tau_{i}\right)$.

We shall say that the solution $y(t)$ of (2.1) belongs to $\varepsilon$-neighbourhood of the integral set $M$ for $t \geq t_{0}$ if there exists a sequence $\left\{\theta_{i}\right\}, i=1,2, \ldots, \theta_{i} \in \Lambda_{i}$, such that $\left|\theta_{i}^{y}-\theta_{i}\right|<\varepsilon$ and $\varrho\left(y\left(t, t_{0}, y_{0}\right), M(t)\right)<\varepsilon$ for $t \geq t_{0}, t \notin \bigcup_{i=1}^{\infty}\left(\theta_{i}-\varepsilon, \theta_{i}+\varepsilon\right)$ (here, $\theta_{i}^{y}, i=1,2, \ldots$, are points of discontinuity of the solution $y(t)$ and $\varrho(y, M(t))$ is a distance between $y$ and the set $M(t))$.

With every point $\left(t_{0}, y_{0}\right) \in \mathbb{R}_{+} \times \mathbb{R}^{n}$, we associate the set $M_{y_{0}}\left(t_{0}\right)$ as follows:

$$
M_{y_{0}}\left(t_{0}\right)= \begin{cases}M\left(t_{0}\right) & \text { if } t_{0} \notin \bigcup_{i=1}^{\infty} \Lambda_{i}, \\ M_{i}\left(t_{0}\right) & \text { if } t_{0} \in \Lambda_{i},\left(t_{0}, y_{0}\right) \in \Omega_{, i} \\ M_{i+1}\left(t_{0}\right) & \text { if } t_{0} \in \Lambda_{i},\left(t_{0}, y_{0}\right) \in \Omega_{i+1} .\end{cases}
$$

We give the following definitions of stability and asymptotic stability of an integral set [7].

The integral set $M$ of system (2.1) is stable if for any $\varepsilon>0$ and $t_{0} \in \mathbb{R}_{+}$there exists such a number $\delta=\delta\left(\varepsilon, t_{0}\right)>0$ that, for any solution $y(t)=y\left(t, t_{0}, y_{0}\right)$ of system (2.1), $\varrho\left(y_{0}, M_{y_{0}}\left(t_{0}\right)\right)<\delta$ implies that $y(t)$ belongs to the $\varepsilon$-neighbourhood of the integral set $M$ for $t \geq t_{0}$ (it is assumed that $t_{0} \neq \theta_{i}^{y}$ ).

The stable integral set $M$ of system (2.1) is asymptotically stable if there exists such a number $\delta_{0}>0$ that for any solution $y(t)=y\left(t, t_{0}, y_{0}\right)$ of (2.1) that satisfies the inequality $\varrho\left(y_{0}, M_{y_{0}}\left(t_{0}\right)\right)<\delta_{0}$, the following holds: $\lim _{t \rightarrow \infty} \varrho(y(t), M(t))=0$. 
These definitions of the stability properties of the integral set of the impulsive system (2.1) agree with the corresponding definitions of stability properties of a single discontinuous solution of $(2.1)[\mathbf{1 , 2}]$.

There are various ways for the investigation of stability of integral sets for impulsive systems. One of them consists of the reduction of the problem to the consideration of a simpler case impulsive system - a system with impulses at fixed times.

Suppose that, in (2.1), the functions $f(t, x)$ and $I_{i}(x)$ satisfy the conditions

$$
\|f(t, x)-f(t, y)\|+\left\|I_{i}(x)-I_{i}(y)\right\| \leq K\|x-y\|
$$

uniformly with respect to $t \in \mathbb{R}_{+}, i=1,2, \ldots$, for all $x, y \in \mathbb{R}^{n}$, and

$$
\sup _{t \geq 0}\|f(t, 0)\|+\sup _{i \geq 1}\left\|I_{i}(0)\right\|<+\infty
$$

Suppose that, for $x \in B_{h}$, the equations of surfaces of discontinuity of solutions can be written in the form

$$
t=t_{i}(x)=\tau_{i}+\tau_{i}(x), i=1,2, \ldots,
$$

and the following conditions are satisfied:

$$
\tau_{i+1}-\tau_{i} \geq d>0, \quad 0 \leq \tau_{i}(x) \leq l<d, \quad\left|\tau_{i}(x)-\tau_{i}(y)\right| \leq l\|x-y\|, \quad i=1,2, \ldots,
$$

where $l$ is a sufficiently small number.

It is proved in [7] that, under such assumptions, the investigation of stability of integral sets of impulsive differential system (2.1) can be reduced to the investigation of stability of integral set of some differential system with impulses at fixed times. One can point out the system with impulses at fixed times and the integral set $\hat{M}$ (constructed by system (2.1) and the set $M$ ) such that the stability (resp., asymptotic stability) of $\hat{M}$ for constructed system implies the stability (resp., asymptotic stability) of the set $M$ for system (2.1). Note that definitions of stability properties of sets in this particular case of impulsive system coincide with well-known definitions for ordinary differential equations [8]. As it was mentioned, investigation of a set's stability for the systems with fixed times of impulses is simpler than in the general case of impulsive system.

To illustrate the possible character of such conditions we adduce one variant of sufficient conditions for stability (asymptotic stability) of an integral set of an impulsive differential system with fixed time moments sequence of impulse action [7].

Consider the system

$$
\begin{aligned}
& \frac{d x}{d t}=F(t, x), t \neq \tau_{i}, \\
& \Delta x_{\mid t=\tau_{i}}=J_{i}(x),
\end{aligned}
$$

where $x \in \mathbb{R}^{n}, t \in \mathbb{R}_{+}, i=1,2, \ldots, 0=\tau_{0}<\tau_{1}<\ldots, \tau_{i} \rightarrow \infty$ as $i \rightarrow \infty$.

As above, assume that system (2.2) satisfies the conditions of existence and uniqueness of solutions, and that any solution of system (2.2) is defined on $\left[t_{0},+\infty\right)$. 
Let $M$ be an integral set of system (2.2). As above, suppose that, for each $t \in \mathbb{R}_{+}$, the section $M(t)$ is not empty and is contained in a compact set $Q \subset \mathbb{R}^{n} ; Q_{\varepsilon_{0}} \subseteq B_{h}$.

Consider a continuously differentiable function $V(t, x)$ defined in the domain $Z=$ $\left\{(t, x): t \in \mathbb{R}_{+}, x \in Q_{\varepsilon_{0}}\right\}$ and possessing the properties

$$
\begin{gathered}
V(t, x)=0,(t, x) \in M ; \quad V(t, x)>0,(t, x) \notin M ; \\
V(t, x) \geq a(\varrho(x, M(t))),
\end{gathered}
$$

where $a(s)(s \geq 0)$ is a continuous increasing function, $a(0)=0$.

Assume that there exists $\mu>0\left(0<\mu<\varepsilon_{0}\right)$ such that, for $x \in Q_{\mu}$, one has $x+J_{i}(x) \in Q_{\varepsilon_{0}}, i=1,2, \ldots$

Consider, for example, the case where the sequence $\left\{\tau_{i}\right\}, i=1,2, \ldots$, satisfies the condition $\tau_{i+1}-\tau_{i} \geq \theta, \theta>0$.

Theorem 6. (A) Let there exist a continuously differentiable function $V(t, x)$ satisfying conditions (2.3) in the domain $Z$ and such that

$$
\begin{gathered}
\frac{\partial V(t, x)}{\partial t}+\left\langle\operatorname{grad}_{x} V(t, x), F(t, x)\right\rangle \leq-\varphi(V(t, x)), \\
V\left(\tau_{i}, x+J_{i}(x)\right) \leq \psi\left(V\left(\tau_{i}, x\right)\right), i=1,2, \ldots
\end{gathered}
$$

where $\varphi(s), \psi(s) \in K_{0}$. If there exists a positive number $a_{0}$ such that, for an arbitrary $a \in\left(0, a_{0}\right]$,

$$
\int_{a}^{\psi(a)} \frac{d s}{\varphi(s)} \leq \theta
$$

then the set $M$ is stable with respect to system (2.2).

(B) Let there exist a continuously differentiable function $V(t, x)$ satisfying in the domain $Z$ conditions (2.3), (2.4), (2.5).

If there exist positive numbers $a_{0}$ and $\gamma$ such that

$$
\int_{a}^{\psi(a)} \frac{d s}{\varphi(s)} \leq \theta-\gamma
$$

for all $a \in\left(0, a_{0}\right]$, then the set $M$ is asymptotically stable with respect to system (2.2).

The form of these conditions is similar to conditions quoted above for the stability of an invariant set of system (1.1) (Theorem 3).

It must be mentioned that problems of sets' stability for impulsive differential equations were studied by many authors, see, e. g., [9-13].

The work [9] studies sufficient conditions of stability, asymptotic stability, instability, and global stability of the trivial invariant torus of the system

$$
\frac{d \varphi}{d t}=a(\varphi), \quad \frac{d x}{d t}=f(\varphi, x),\left.\quad \Delta x\right|_{t=\tau_{i}}=I(\varphi, x),
$$

where $a(\varphi) \in C_{\text {Lip }}\left(\mathscr{T}_{m}\right)$, the functions $f(\varphi, x)$ and $I_{i}(\varphi, x)$ are defined in the domain $Z=\left\{\varphi \in \mathscr{T}_{m}, x \in \bar{J}_{h}\right\}$ (here, $\bar{J}_{h}=\left\{x \in \mathbb{R}^{n}:\|x\| \leq h, h>0\right\}$ ), are $2 \pi$-periodic with 
respect to each of their variables $\varphi_{v}, v=1, \ldots, m$, and, moreover, $f(\varphi, 0) \equiv 0$, $I(\varphi, 0) \equiv 0$ for all $\varphi \in \mathscr{T}_{m}$. The sequence $\left\{\tau_{i}\right\}$ is assumed to be such that $\tau_{i}>$ $\tau_{i-1}, i=1,2, \ldots$, and $\lim _{i \rightarrow \infty} \tau_{i}=\infty$.

The paper [9] also considers stability conditions for the trivial invariant torus of the burst dynamical system

$$
\frac{d \varphi}{d t}=a(\varphi), \quad \frac{d x}{d t}=f(\varphi, x),\left.\quad \Delta x\right|_{\varphi \in \Gamma}=I(\varphi, x),
$$

where $\Gamma$ is a subset of the torus $\mathscr{T}_{m}$ (a manifold of dimension $m-1$ ).

It is important to note that, for solving the problem on stability of an invariant torus, it is sufficient to consider the functions $V(\varphi, x), V(\varphi, I(\varphi, x))$, and $\frac{d V}{d t}$ in the region $Z_{\Omega}=\left\{\varphi \in \Omega, x \in \bar{J}_{h}\right\}$ only, and not everywhere in the region $Z$ (here, $\Omega=\bigcup_{\varphi \in \mathscr{T}_{m}} \Omega_{\varphi} \subset \mathscr{T}_{m}, \Omega_{\varphi}$ is the $\omega$-limit set of the positive half-trajectory of the solution $\varphi_{t}(\varphi), \varphi \in \mathscr{T}_{m}, t \in[0,+\infty)$ of the first equation of system (2.6) or (2.7)).

In [10], the stability of a set $M \subset \mathbb{R}_{+} \times \mathbb{R}^{n}$ is investigated for an impulsive system of the general form (2.1) and, in [13], for the system

$$
\begin{gathered}
\frac{d x}{d t}=f(t, x(t), x(t-h)), t>t_{0}, t \neq \tau_{k}(x(t)), \\
x(t)=\varphi_{0}(t), t \in\left[t_{0}-h, t_{0}\right], \\
\left.\Delta x(t)\right|_{t=\tau(x(t))}=I_{k}(x(t)), t>t_{0}, \quad k=1,2, \ldots
\end{gathered}
$$

Note that the definitions of stability of a set $M \subset \mathbb{R}_{+} \times \mathbb{R}^{n}$ for the system (2.1) used in $[\mathbf{1 0}, \mathbf{1 3}]$ correspond to the definitions of stability properties of sets for ordinary differential equations (without impulses) [8] and agree with the definitions of stability properties for the trivial (continuous) solution of an impulsive equation [1,2].

The methods of investigation of the stability of sets in the papers cited above are based on Lyapunov's direct method and its generalisations. As one can see [11-13], it is also efficient to apply the comparison principle together with the Lyapunov second method.

\section{REFERENCES}

[1] Samoilenko, A. M. and Perestyuk, N. A.: Impulsive Differential Equations, Vishcha Shkola, Kiev, 1987 (in Russian).

[2] Samoilenko, A. M. and Perestyuk, N. A.: Impulsive Differential Equations, World Scientific, Singapore, 1995.

[3] Perestyuk, N. A. and Chernikova, O. S.: On stability of invariant sets of discontinuous dynamical systems, Ukr. Mat. Zh., 53 (2001), No. 1, 78-84; translation in Ukrainian Math. J., 53 (2001), No. 1, 91-98.

[4] Gurgula, S. I. and Perestyuk, N. A.: On the stability of solutions of impulsive systems, Vestnik Kiev. Univ. Matem. Mech., 23, (1981), 33-40 .

[5] Perestyuk, N. A. and Chernikova, O. S.: Stability of solutions of impulsive systems, Ukr. Mat. Zh., 49 (1997), No. 1, 98-111; translation in Ukrainian Math. J., 49 (1997), No. 1, 109-123.

[6] Lakshmikantham, V.: Trends in the theory of impulsive differential equations, Proc. Internat. Conf. on the Theory and Applications of Differential Equations, Ohio University, 1988, pp. 76-87. 
[7] Perestyuk, N. A. and Chernikova, O. S.: On the stability of integral sets of impulsive differential sestems, Miskolc Mathematical Notes, 2 (2001), No. 1, 49-60.

[8] Yoshizawa, T.: Stability of sets and perturbed systems, Funkcialaj Ekvacioj, 5 (1962), 31-69.

[9] Perestyuk, N. and Doudzianiy, S.: Stability of invariant toruses of impulsive systems, Appl. of Math. in Engineering and Economics, Heron Press, Sofia, 2000, 28-32.

[10] Kulev, G. K. and Bainov, D. D.: Global stability of sets for impulsive differential systems by Lyapunov's direct method, Comput. Math. Appl., 19 (1990), No. 2, 17-28.

[11] Liu, X. and Pirapakaran, R.: Global stability results for impulsive differential equations, Appl. Analysis, 33 (1989), 87-102.

[12] Bainov, D. D. and Stamova, I. M.: Stability of sets for impulsive differential-difference equations with variable impulsive perturbations, Comm. Appl. Nonlinear Anal., 5 (1998), No. 1, 69-81.

[13] Sтамоv, G. T.: Stability of moving invariant manifold for impulsive differential equations, J. Techn. Univ. Plovdiv Fundam. Sci. Appl., 7 (1999), 99-107.

\section{Authors' Addresses}

\section{Giovanni Matarazzo:}

Department of Computer Engineering and Applied Mathematics, Salerno University, via Ponte Don Melillo, 84084 Fisciano (SA), Italy

E-mail address: matarazz@diima.unisa.it

\section{Nikolai A. Perestyuk:}

Department of Mechanics and Mathematics, Kiev National Taras Schevchenko University, VoloDimiRSKA 64, 01033 KIEv, UKRAINE

E-mail address: pmo@univ.kiev.ua

\section{Olga S. Chernikova:}

Department of Mechanics and Mathematics, Kiev National Taras Schevchenko University, VoloDimiRSKa 64, 01033 Kiev, UKRAINE

E-mail address: pmo@univ.kiev.ua 